\title{
Single Particle Nanoplasmonic Sensing in Individual Nanofluidic Channels
}

\author{
Joachim Fritzsche, ${ }^{\dagger}$ David Albinsson, ${ }^{\dagger}$ Michael Fritzsche, Tomasz J. Antosiewicz, ${ }^{\S}$ Fredrik Westerlund, ${ }^{*},+$ \\ and Christoph Langhammer*, ${ }^{*}$
}
${ }^{\dagger}$ Department of Physics and ${ }^{\ddagger}$ Department of Biology and Biological Engineering, Chalmers University of Technology, 41296 Göteborg, Sweden
${ }^{\S}$ Centre of New Technologies, University of Warsaw, 02-097 Warsaw, Poland

\section{Supporting Information}

\begin{abstract}
Nanoplasmonics allows label-free optical sensing and spectroscopy at the single nanoparticle level by exploiting plasmonic excitations in metal nanoparticles. Nanofluidics offers exclusive possibilities for applying and controlling fluid flow and mass transport at the nanoscale and toward nanosized objects. Here, we combine these two concepts in a single device, by integrating single particle nanoplasmonic sensing with nanofluidics using advanced nanofabrication. The developed devices enable on-chip referenced parallel single particle nanoplasmonic sensing

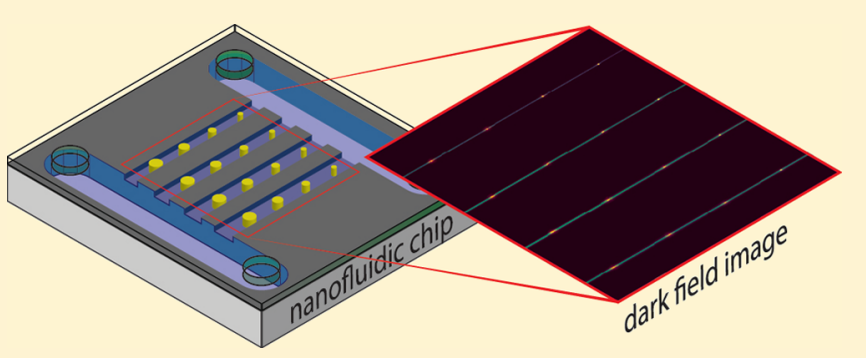
inside multiple individual nanofluidic channels with dimensions down to the $100 \mathrm{~nm}$ range. Beyond detailed discussion of the nanofabrication, general device characterization, and parallelized single particle plasmonic readout concepts, we demonstrate device function on two examples: (i) in situ measurements of local buffer concentrations inside a nanofluidic channel; (ii) real time binding kinetics of alkanethiol molecules to a single plasmonic nanonatenna sensor in a single nanochannel. Our concept thus provides a powerful solution for controlling mass transport to and from individual (plasmonic) nanoparticles, which in a long-term perspective offers unique opportunities for label-free detection of analyte molecules at low concentrations and for fundamental studies of fluids in extreme confinement.
\end{abstract}

KEYWORDS: Single particle plasmonic sensing, nanofluidics, nanochannel, parallelized single particle plasmonic readout, dark-field scattering spectroscopy, nanofabrication

$\mathrm{N}$ anofluidics has evolved in the footsteps of microfluidics as a natural step to further downsize the control of fluids to the nanoscale. ${ }^{1}$ In nanofluidic systems, fluids can be confined to volumes smaller than their characteristic physical scaling lengths. This gives rise to new fascinating phenomena, ${ }^{2-6}$ and in the field of nanofluidics these effects are explored to study individual (bio)molecules like DNA, ${ }^{7}$ enzymes, ${ }^{3,8}$ and proteins, ${ }^{9-11}$ as well as for investigation of immunochemical reactions. ${ }^{12}$ Nanofluidic systems thus offer exclusive possibilities for applying and controlling fluid flow and mass transport at the nanoscale, as well as toward and away from nanosized objects. At the same time, at the nanometric length scale at hand, metallic nanoparticles show optical properties that are distinct from the bulk. This is due to the excitation of localized surface plasmon resonances (LSPR) when the particles interact with light and act as optical nanoantennas. ${ }^{13}$ The polarization of electrons in the nanoparticle by the incoming photons creates locally enhanced electric fields, which make plasmonic nanoantennas sensitive probes for the detection of changes in their local surroundings, for example, the refractive index of a fluid or the binding of molecular species to the nanoantenna surface. ${ }^{14}$ These effects have been widely explored in nanoplasmonic bio- and chemosensing. ${ }^{15-17}$ Within these research areas, the detection of discrete binding events on single nanostructures has positioned itself as one of the ultimate goals. As a consequence, numerous research groups have taken on this challenge and have presented different solutions based on label-free refractometric plasmonic sensing using predominantly dark-field scattering spectroscopy ${ }^{18}$ for detection of individual molecular binding events to a single plasmonic nanosensor. ${ }^{19-27}$ At the same time, the combination of plasmonics and Raman spectroscopy has led to advances in few-molecule investigations by concentrating light in plasmonic hot spots. ${ }^{28,29}$ However, despite these significant conceptual advances one critical issue remains, namely that molecules are free to diffuse away from the plasmonic surface if no specific measures are taken. This means that when the concentration of the analyte is low and the size of the sensor element is in the range of a few tens of nanometers, the accumulation time for the detection of a few molecules dispersed in a solution is on the scale of days. ${ }^{30,31}$

Received: October 1, 2016

Revised: November 14, 2016

Published: November 15, 2016 


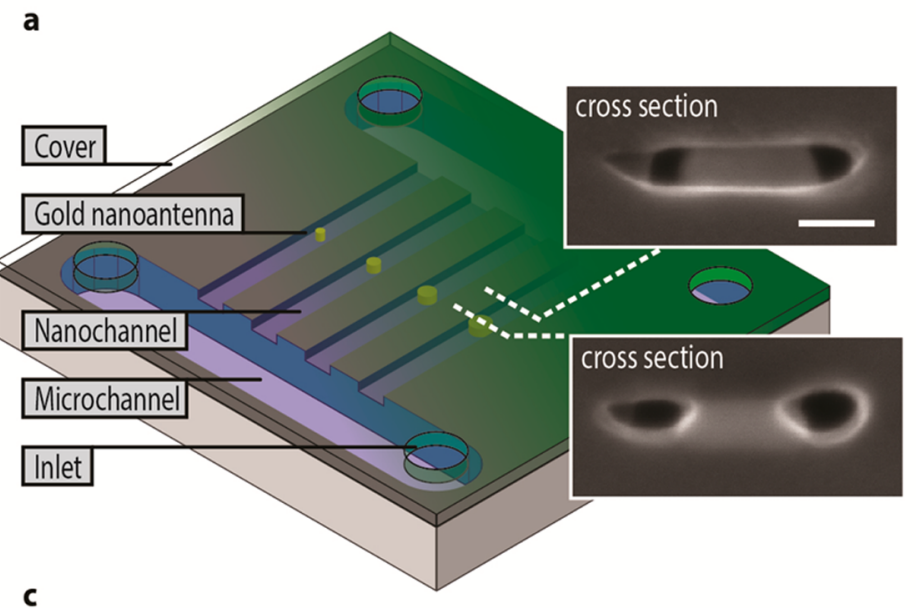

b
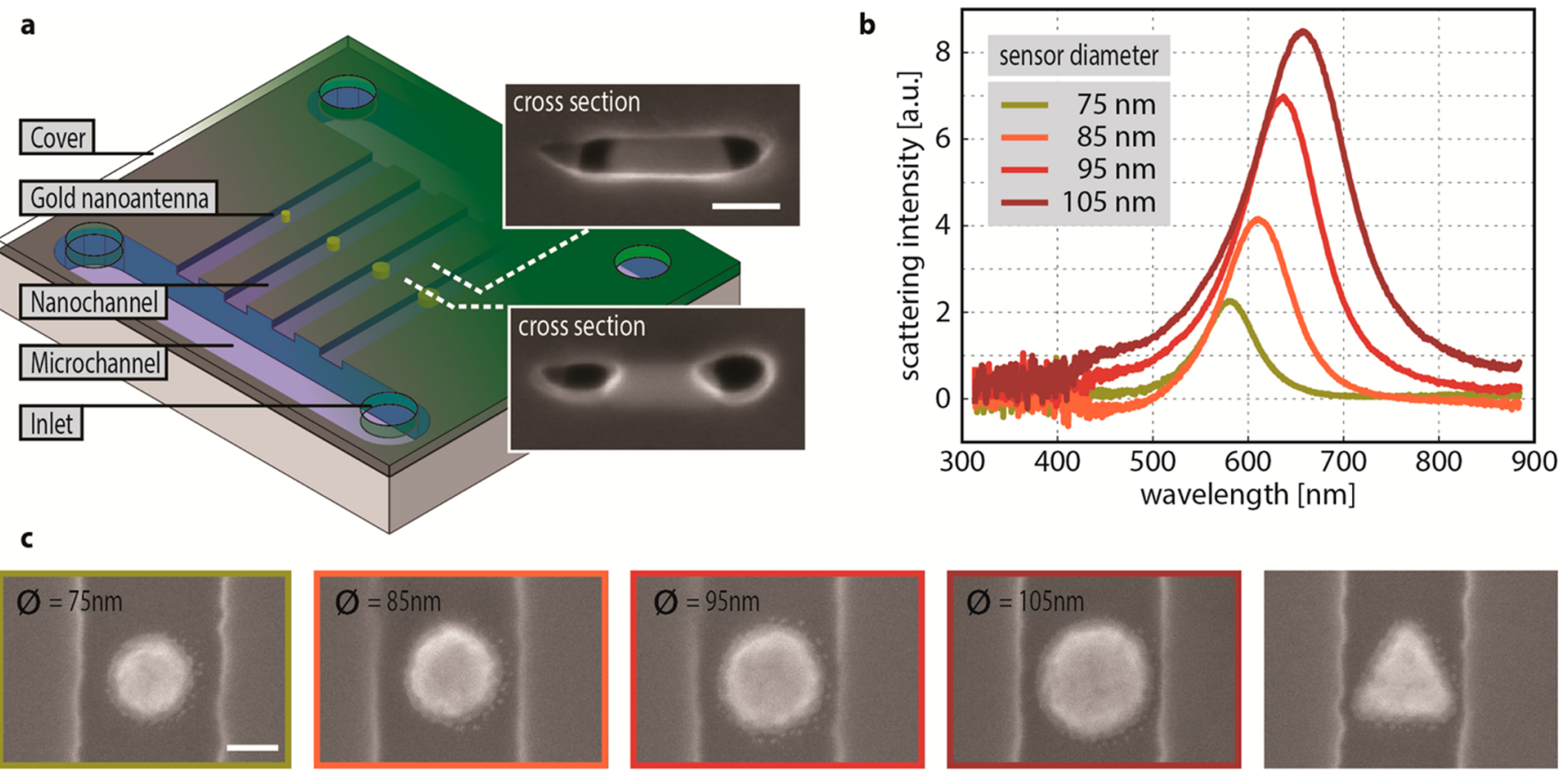

Figure 1. Schematic device layout together with scanning electron microscopy (SEM) characterization and corresponding optical response of single $\mathrm{Au}$ nanoantennas inside a single nanochannel. (a) Schematic depiction of a typical nanofluidic chip (not to scale) used for single particle nanoplasmonic sensing in individual nanochannels. It is comprised of two microchannels of $50 \mu \mathrm{m}$ width and $1.5 \mu \mathrm{m}$ depth, connected to an array of nanochannels of $120 \mathrm{~nm}$ width, $30 \mathrm{~nm}$ depth, and $350 \mu \mathrm{m}$ length, integrated with single plasmonic nanoantennas with different size and shape. The scanning electron micrographs taken after bonding of the lid show cross sections through a nanochannel (top) close to an integrated disk-shaped gold nanoantenna and (bottom) through a gold nanoantenna. The scale bar is $50 \mathrm{~nm}$. Note that in such an arrangement it becomes possible to probe the entire volume of fluid flowing past the antenna, that is, any fluid and/or molecule flowing through the nanochannel must pass within the plasmonic field decay length, provided the gap between the nanoantenna and the channel wall is on the order of ca. $30-50 \mathrm{~nm}$. $^{33}$ (b) Dark-field scattering spectra and (c) corresponding SEM micrographs (taken prior to bonding of the lid) of individual disk-shaped gold nanoparticles with different diameters measured in water-filled nanochannels with $120 \mathrm{~nm}$ width and $30 \mathrm{~nm}$ depth. The scattering spectrum of the nanotriangle is shown in Figure S2 in the SI. The scale bar is $50 \mathrm{~nm}$.

In view of the above, it is of critical importance to address the problem of controlling nanoscale mass transport in the context of single particle nanoplasmonic sensing and to find ways to guide analyte molecules directly toward single plasmonic nanoparticles and their hotspots. To this end, to our knowledge the only reported approach is the work by De Angelis et al., who used a superhydrophobic surface to concentrate analyte on a plasmonic Raman probe. ${ }^{32}$ Here, we present a conceptually different approach based on the central idea of merging nanofluidics with single particle nanoplasmonic sensing and spectroscopy. Specifically, by using advanced nanofabrication techniques, we manufacture nanofluidic devices where individual plasmonic nanoparticles are placed with high precision inside individual nanofluidic channels, and which allow single particle optical spectroscopy based on dark-field scattering (Figure 1). We highlight that it in such an arrangement becomes possible to probe the entire volume of fluid flowing past the antenna, that is, any fluid and/or molecule flowing through the nanochannel must pass within the plasmonic field decay length, provided the gap between nanoantenna and channel wall is less than ca. $30-50 \mathrm{~nm}^{33}$

Beyond the detailed discussion of the nanofabrication of these devices and their characterization in terms of bulk refractive index sensitivity and sensing figure of merit (FoM), we demonstrate both monochromatic-illumination-based readout of multiple nanochannels/single particles simultaneously, and on-chip signal referencing to significantly improve the signal-to-noise ratio and long-term stability of the mono- chromatic readout. Finally, we also demonstrate the attainable single nanoparticle sensing function inside nanofluidic structures on two examples: (i) in situ measurements of local buffer concentrations inside a nanofluidic channel; (ii) real time binding kinetics of alkanetiol molecules to a single plasmonic nanoantenna sensor in a single nanochannel.

As the first characterization step of our device we determine how the bulk refractive index sensitivity of gold nanoantennas of different size $(75,85,95$, and $105 \mathrm{~nm}$; Figure $2 \mathrm{a})$ is affected by the proximity of the nanochannel walls. This is important because it constitutes a feature unique to our devices where the dimensions of the fluidic system are comparable to those of the plasmonic sensor particle. The LSPR peak position of the nanoparticles in water as a function of particle size, and sorted by the width of the corresponding hosting nanochannel, is depicted in Figure 2b. As expected, the LSPR frequency is redshifted for increasing nanoparticle size, irrespective of nanochannel width. To derive the bulk refractive index sensitivity, we repeatedly (4 times) filled the fluidic system with water and ethylene glycol, respectively, to vary the refractive index, $n$, of the fluid in the channel from $n=1.33$ (water) to $n=1.43$ (ethylene glycol); see Methods and SI for details. From the measured shift in LSPR peak position of the nanoantennas, $\Delta \lambda$, induced by changing $n$ of the fluid, we determined the bulk refractive index sensitivity, $\Delta \lambda / \Delta \mathrm{n}$ (Figure 2c) and the FoM of the individual antennas in the different channels (Figure $2 \mathrm{~d}$ ). The FoM is defined as the resonance shift $\Delta \lambda$ upon a change in the refractive index $n$ of the surrounding medium normalized 
a

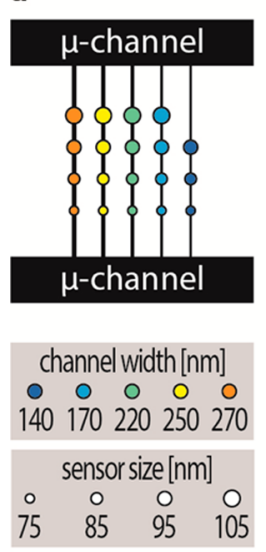

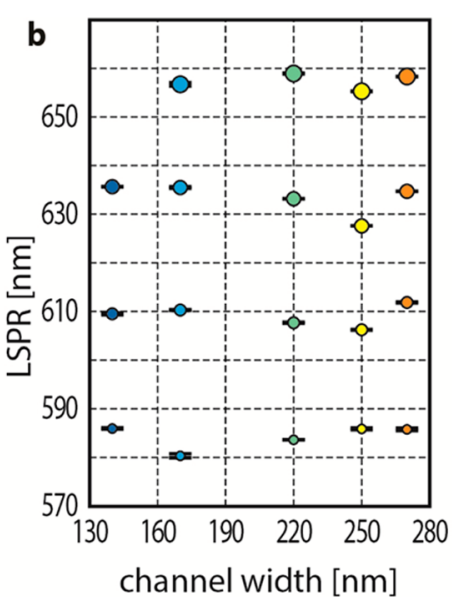

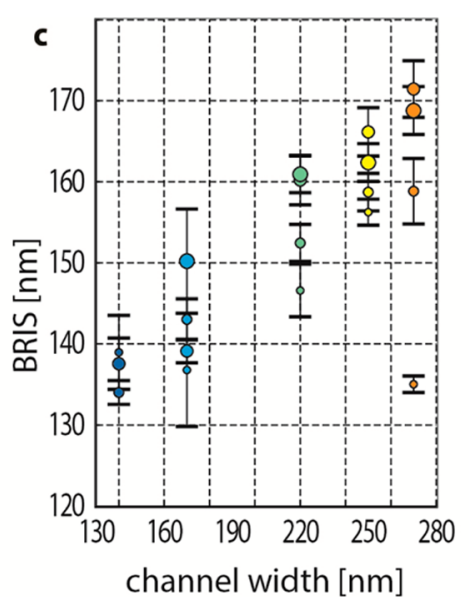

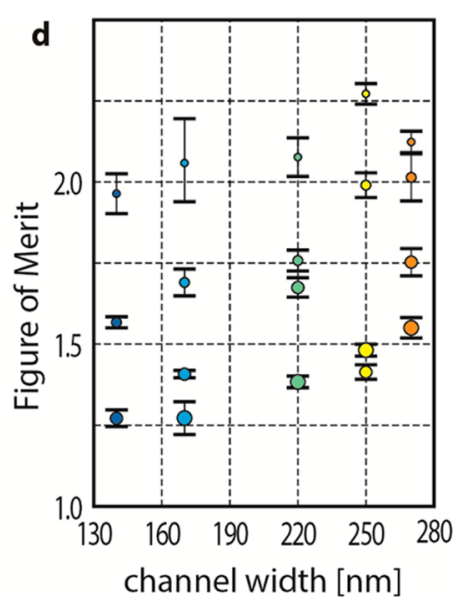

Figure 2. Bulk refractive index sensitivity (BRIS) and figure of merit (FoM) analysis for nanochannels with different widths. (a) Schematic depiction of the device used together with polychromatic illumination for experimental assessment of bulk refractive index sensitivity and figure of merit, together with the legend for the subsequent figure panels. (b) LSPR wavelength of individual nanoantennas with different diameters inside waterfilled nanochannels with different widths. As expected, the LSPR frequency is red-shifted for increasing nanoparticle size, independent of nanochannel width. The minor differences in frequency for nanoparticles of nominally identical size are caused because their shape may be slightly altered during the fusion bonding step of the lid at $550{ }^{\circ} \mathrm{C}$, which induces recrystallization. Error bars indicate standard deviation around the mean derived from four consecutive measurements of each particle. (c) Bulk refractive index sensitivity for differently sized nanoantennas in nanochannels with different widths. We find higher bulk refractive index sensitivity for larger nanodisks resonating at lower photon energies in channels of the same dimensions, which is in agreement with the literature for open surfaces. Increasing the channel width leads to an increase in bulk refractive index sensitivity for all nanodisk sizes due to a smaller fraction of the sensing volume being occupied by the channel walls (see also FDTD simulation in Figure S8 in SI) and thus not being susceptible for a change of the fluid refractive index inside the channel. The small deviation from the general trend by some of the particles is explained by the fact that their shape may be altered during fusion bonding. (d) Figure of merit as a function of nanoantenna size in nanochannels with different widths. The measured values range from $\sim 1$ to 2.5 and are larger for smaller nanoantennas, which agrees well with the literature for noncoupled individual plasmonic nanoantennas. The dependence of the FoM on the nanochannel width is the same as for the bulk refractive index sensitivity, that is, slightly larger FoM for wider nanochannels.

by the resonance line width. In agreement with similar studies of the bulk refractive index sensitivity of plasmonic nanoantennas on open surfaces, ${ }^{33-36}$ we find higher bulk refractive index sensitivity for larger nanodisks resonating at lower photon energies, for constant channel width. Some deviation from the general trend by some of the particles is explained by the fact that their shape may be altered during the fusion bonding step of the lid $\left(550^{\circ} \mathrm{C}\right)$, thus, for example, modulating the bulk refractive index sensitivity due to the formation of sharp features due to recrystallization. All in all the above indicates that the general and well-established concepts and sensitivity scaling with plasmonic nanoantenna size and shape are also valid for single plasmonic nanoantennas confined inside a nanofluidic structure.

Having established this fact, it is interesting to investigate how the bulk refractive index sensitivity depends on the nanochannel width. As a general trend, we find that increasing the channel width leads to an increase in bulk refractive index sensitivity for all nanodisk sizes (Figure 2c). This can be understood as that for narrower channels a larger fraction of the sensing volume defined by the enhanced near field is occupied by the channel walls and thus not susceptible for a change of $n$ inside the channel. This is also confirmed by finite-difference time domain (FDTD) simulations summarized in Figure S8 in the SI. Again, also in this case, the deviation of some particles from the general trend may be explained by the variations in nanoantenna shape induced by the fusion bonding step.

For the analogue analysis of the FoM in Figure 2d, we find a FoM that ranges between 1 and 2.5, which agrees well with the literature for noncoupled individual plasmonic nanoantennas, ${ }^{37}$ and as a general trend we obtained a higher FoM for smaller nanodisk size. The reason for the higher FoM of the smaller structures is their significantly narrower line-width (see Figures S5 and S6) due to reduced radiation damping. ${ }^{38}$ The dependence of the FoM on the nanochannel width is the same as for the bulk refractive index sensitivity, that is, slightly larger FoM for wider channels. This is expected since the peak line width does not exhibit clear nanochannel-width dependence (see Figure S6), which renders the bulk refractive index sensitivity contribution the dominating factor defining the FoM. As a consequence of this analysis, below we use the smallest $(75 \mathrm{~nm})$ nanoparticles in channels of $140 \mathrm{~nm}$ width to illustrate the attainable single nanoparticle sensing function inside nanofluidic structures on two specific examples.

To demonstrate the sensing, referencing and parallel readout functionality of our devices, we use a chip design as depicted in Figure 3a. Moreover, we implement a new monochromatic illumination scheme to eliminate unwanted light scattering from the nanochannel walls, as discussed in detail in the SI. The nanofluidic chip is comprised of an array of seven nanochannels, each decorated with a single nanoantenna. The first six channels are connected to a microchannel on both ends and act as "sample channels", because fluid flow can be established through them by applying a pressure gradient via pressurizing the microfluidic system. The nanoantennas in the channels were designed such that the first three antennas had the same size $(80 \mathrm{~nm})$. Their LSPR frequency was tailored such that the corresponding scattering peak exhibits its inflection point on the right flank as close as possible to the $633 \mathrm{~nm}$ wavelength of the $\mathrm{HeNe}$ laser used for illumination (inset in Figure 3c) according to the monochromatic illumination scheme presented in Figure S4d in the SI. The other three nanoantennas were made larger $(84,88$, and $92 \mathrm{~nm})$ to red shift the LSPR, and for the largest one the LSPR peak occurred very close to 
a

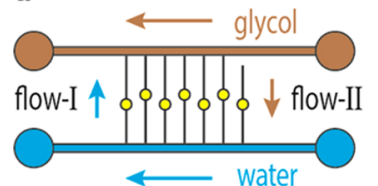

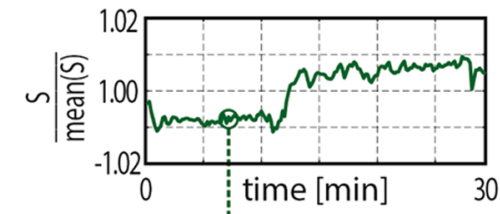

C

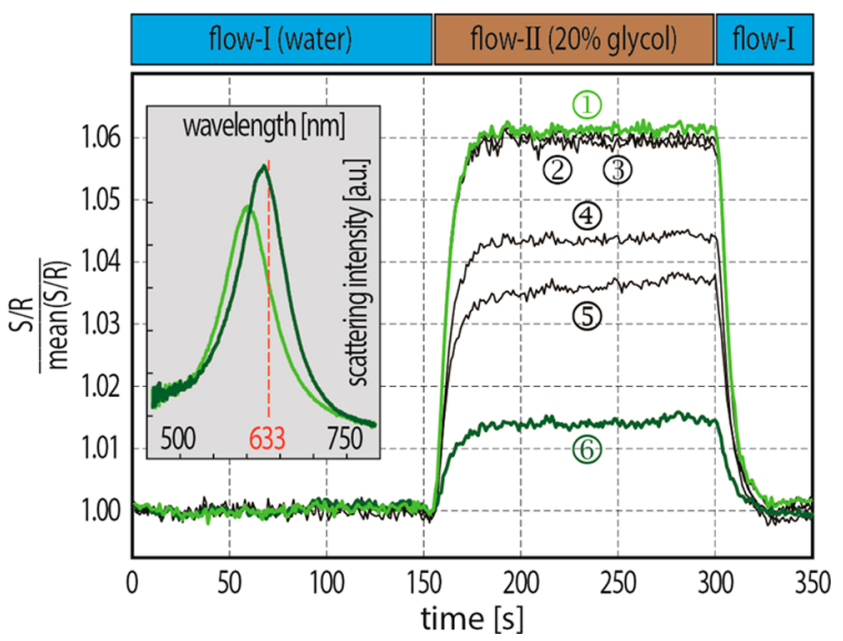

Figure 3. Parallel monochromatic-illumination-based refractive index sensing in multiple nanochannels. (a) Schematic of the device used for on-chip referenced parallelized single particle sensing using monochromatic HeNe laser illumination at $633 \mathrm{~nm}$ (see SI for details). It is comprised of an array of seven nanochannels, each decorated with a single nanoantenna. The first six channels act as "sample channels" through which fluid flow can be established. The nanoantennas 1-3 have the same size $(80 \mathrm{~nm})$, which is tailored such that their LSPR scattering peaks have their inflection points on the right flank as close as possible to the $633 \mathrm{~nm}$ wavelength of the HeNe laser (light green curve in the inset in panel c). The other three nanoantennas are larger (84-92 nm) and thus exhibit a red-shifted LSPR. For the largest one (6), the LSPR peak basically occurs at the irradiated laser wavelength of $633 \mathrm{~nm}$ (inset in panel c). The seventh "dead end" nanochannel is only connected to one side of the microfluidic system and can therefore be used as on-chip reference because the nanoantenna in this channel will remain immersed in the same fluid for the entire experiment (if the flow in the lower microchannel is maintained as indicated by the arrow) and thus experience a constant environment. (b) CCD image of the seven nanoantennas illuminated at $633 \mathrm{~nm}$. The particles are revealed as bright dots. The top panel shows the scattering signal normalized by its mean value for the nanoantenna located in channel six, measured during $30 \mathrm{~min}$ of water flowing through the nanochannel. The bottom panel shows the same data normalized by the reference signal obtained simultaneously, thus correcting for intensity noise of the laser and yielding almost perfectly flat single particle readout over the entire $30 \mathrm{~min}$ duration of the experiment. Note that the noise level of the measurement is dominated by the readout noise of the used CCD camera. (c) Parallel and online referenced measurement of the fluid exchange from water to 20 wt \% aqueous ethylene glycol solution in six nanochannels. The three nominally identical nanoantennas with inflection points overlapping with the illumination wavelength (red dashed lines) exhibit the largest and basically identical absolute response. The signal obtained from the larger nanoantennas is smaller and becomes minimal for the antenna where the peak maximum basically overlaps with the laser line (dark green curve). Note that we used different integration times for each data point, that is, $10 \mathrm{~s}$ in (b) and $1 \mathrm{~s}$ in (c), respectively. This is the reason for the 10 times larger noise in (c) and gives rise to a signal-to-noise ratio of 60 in (c).

the irradiated laser wavelength of $633 \mathrm{~nm}$ (inset in Figure 3c). The seventh nanochannel is designed as a "reference channel" and is therefore only connected to one side of the microfluidic system. In this way, the Au particle in this nanochannel will be immersed in the same fluid for the entire experiment and can be utilized as an on-chip reference to continuously correct for nonspecific signal changes such as light-source intensity fluctuations. This is of particular importance in the present case, where we use a scattering intensity change at a single wavelength as the readout to demonstrate the monochromatic readout concept, Figure $3 \mathrm{~b}$. For the experiment, the CCD is operated in imaging mode (see Methods for details) and reveals the nanoantennas in the nanochannels illuminated at $633 \mathrm{~nm}$ as bright dots with the reference particle furthest to the right. The top panel shows the scattering signal (normalized by its mean value) for the nanoantenna located in channel six, measured during $30 \mathrm{~min}$ as water flows through the nanochannel. The signal intensity fluctuates randomly and also exhibits a distinct step at ca. $11 \mathrm{~min}$. Using the nanoantenna located in the seventh nanochannel as a reference and dividing the scattering signal of the "sample antenna" in channel six by the one obtained from the reference antenna in channel seven, all the unwanted features are completely eliminated and we obtain an almost perfectly flat single particle readout over the entire 30 min duration of our measurement. This is an important step forward for single nanoparticle nanoplasmonic sensing, which often is plagued by unwanted signal fluctuations and long-term drifts. It becomes uniquely available using our nanofluidics concept, which makes it possible to isolate a reference nanoparticle in an inert and constant fluid environment where it will not be exposed to any analyte but still can be kept in the same fluid environment as the sample nanoparticle(s).

We demonstrate this new function specifically in our next experiment, where we simultaneously measure the fluid exchange from water to ethylene glycol in the six sensor channels, using the seventh and constantly water-filled one as reference (Figure 3c). We find good reproducibility for the three nominally identical nanoparticles, which also exhibit the largest absolute response. This is anticipated ${ }^{39}$ because, as evident from the corresponding spectrum shown in the inset of Figure $3 c$, their LSPR scattering peak inflection point occurs close to the $633 \mathrm{~nm}$ laser line. The absolute response obtained from the larger nanoantennas is smaller as the laser line approaches the peak maximum and becomes minimal for the antenna where the peak maximum overlaps with the laser line (green curves in Figure $3 c$ and the inset). The observed maximum signal change in Figure $3 c(0.06)$ corresponds well with the expected value (0.05) considering a bulk refractive index sensitivity of $160 \mathrm{~nm} / \mathrm{RIU}$ (see Figure 2c) and a plasmon line width of $80 \mathrm{~nm}$ (see Figure S6b), while assuming a 


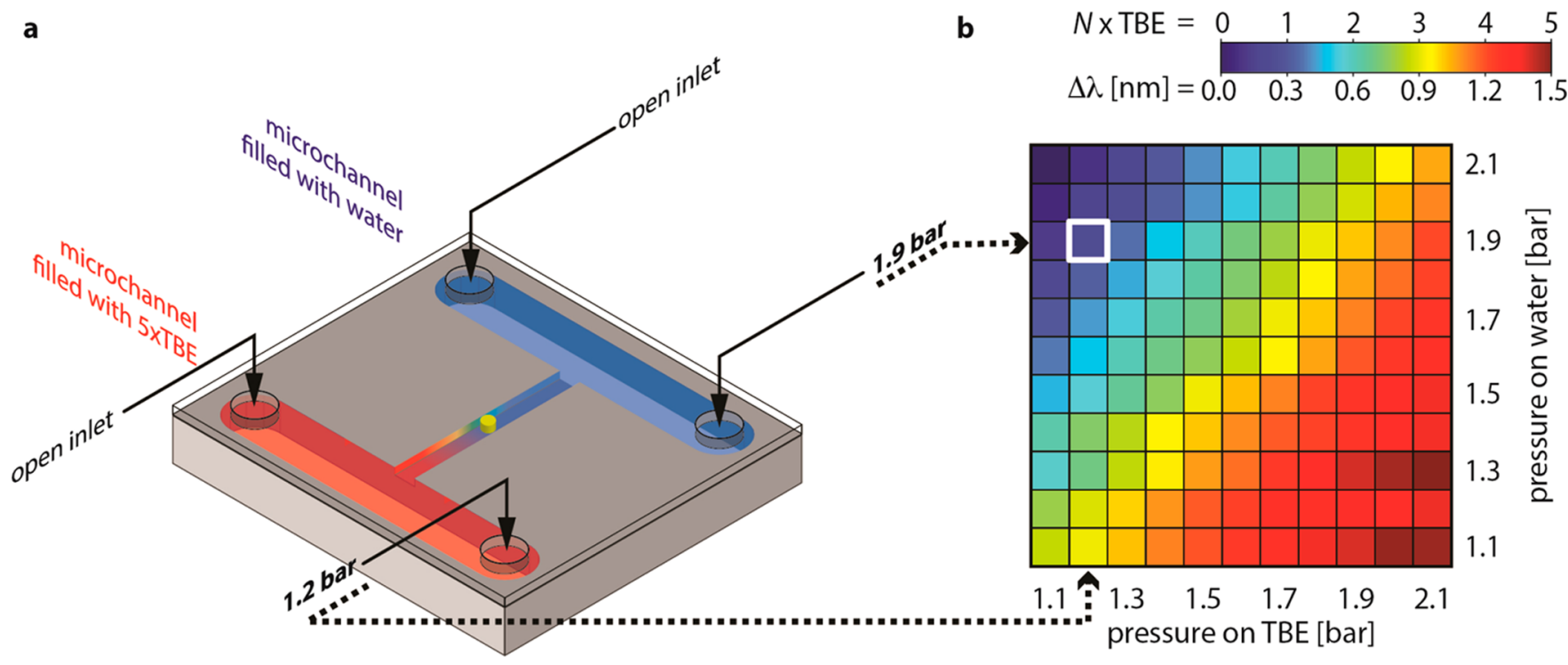

Figure 4. Local readout of buffer concentration inside a nanochannel. (a) Simplified schematic depiction of the nanofluidic device used to manipulate the concentration, $N$, of TBE buffer inside the nanofluidic channel. The two microchannels were filled with $5 \times$ TBE (red) and water (blue), and independent pressures were applied to one inlet of each microchannel. The resulting pressure drop over the nanochannel causes a varying TBE concentration inside it, depending on the applied pressures. (b) The locally measured TBE concentration obtained by reading out the corresponding spectral shift, $\Delta \lambda$, of the LSPR of the individual gold nanoantenna sensor placed in the middle of the nanochannel using polychromatic illumination. The achieved detection limit is $\sigma_{\mathrm{N}} \approx 0.3 \times \mathrm{TBE}$ and can be improved to $\sigma_{\mathrm{N}} \approx 0.08 \times \mathrm{TBE}$ using the monochromatic readout introduced in Figure 3 (see Figure S10 in the SI). The used plasmonic nanoantenna was precalibrated in terms of $\Delta \lambda(N)$ in order to derive the local concentration (see Figure S9 for details). The marked data point corresponds to the situation shown in (a), with pressures of 0.9 and 0.2 bar applied to the microchannels filled with water and $5 \times$ TBE, respectively.

Lorentzian plasmon peak with its inflection point at the laser excitation wavelength.

As the first application example of our device we present in situ measurements of local buffer concentration inside a nanofluidic channel. This particular example is motivated by the fact that nanofluidic devices are frequently used for studying individual biomolecules, such as DNA, under different conditions $^{40-42}$ to correlate their properties with their local chemical and physical environment. A particular challenge in this type of application is to locally assess the applied conditions, for example, temperature, concentration of reagents, or ionic strength, inside the nanofluidic system. Under this aspect we monitor, in real time, the locally changing concentration of TBE buffer ${ }^{43}$ inside a single nanochannel, using traditional nanoplasmonic sensing "peak shift" readout and polychromatic illumination. We use a nanofluidic device comprised of a $140 \mathrm{~nm}$ wide and $30 \mathrm{~nm}$ deep nanochannel and $50 \mu \mathrm{m}$ wide and $1 \mu \mathrm{m}$ deep microchannel (Figure $4 \mathrm{a}$ ). In the experiment, we control the TBE buffer concentration (concentrations of Tris, boric acid, and EDTA are $N \times 89$ $\mathrm{mM}, N \times 89 \mathrm{mM}$ and $N \times 2 \mathrm{mM}$, respectively) inside the nanochannel by filling one of the two connecting microchannels with water and the other one with $5 \times \mathrm{TBE}$, and by then applying different pressures to their inlets to vary the pressure drop across the nanochannels to control flow direction and rate through the nanofluidic system. This is a typical scenario in a nanofluidics experiment when different flows through the channel are sought. The flow velocity increases with increasing pressure drop, supporting or hindering the diffusion of reagents into the nanochannels from the reservoir on the side of high and low pressure, respectively.

Figure $4 \mathrm{~b}$ shows the resulting TBE concentration, $N$, measured locally in the middle of the nanochannel (i.e., at equal distance from both microchannels) using a precalibrated (in terms of translating the plasmon resonance peak shift, $\Delta \lambda$, into TBE concentration; see Figure S8 in the SI) nanoantenna sensor. Clearly, $N$ is very sensitive to the pressures applied on the respective reservoirs. This highlights the importance of the new information that can be obtained by our sensing concept inside nanochannels because as, our measurements show, predicting accurately the resulting local concentrations is nearly impossible even in the case of our relatively simple device with straight channels. In more complex fluidic systems comprising crossings or constrictions, ${ }^{42}$ concentration distributions of reagents will be even more complicated to predict. Thus, in such systems integrated local plasmonic readout can constitute a powerful tool for measuring and verifying targeted specific conditions in situ, to derive correct correlations between (bio)molecule properties and local environment.

To this end it is also interesting to discuss the detection limit of our device (here for $1 \mathrm{~s}$ integration time of the CCD camera). We define the standard deviation of the $\Delta \lambda$ signal as the spectral noise, $\sigma$, and find that $\sigma_{\text {res }} \approx 0.1 \mathrm{~nm}$, in good agreement with similar studies. ${ }^{26,44} \mathrm{We}$ further define the smallest reliably measurable $\Delta \lambda$ signal as $\Delta \lambda_{\text {min }}=\sigma_{\text {res }}=0.1 \mathrm{~nm}$. With $\Delta \lambda$ for $5 \times \mathrm{TBE}$ equal to $1.5 \mathrm{~nm}$ (Figure $4 \mathrm{~b}$ ), we thus derive a detection limit $\sigma_{\mathrm{N}} \approx 0.3 \times \mathrm{TBE}$. Having established this number we also investigate how much it can be improved by using the monochromatic readout alternative introduced above. As shown in Figure S10 in the SI, the spectral noise for the monochromatic readout is 3.5 times smaller for the same CCD settings, which improves the detection limit to $0.08 \times$ TBE. In addition, these numbers can be further improved significantly by choosing a plasmonic structure with higher bulk refractive index sensitivity, for example, a nanorod or other high aspect ratio structures.

As a second application example of our devices we demonstrate the possibility to detect specific binding of 
molecules to a plasmonic antenna inside a nanochannel, Figure 5. Also here we chose polychromatic illumination and the

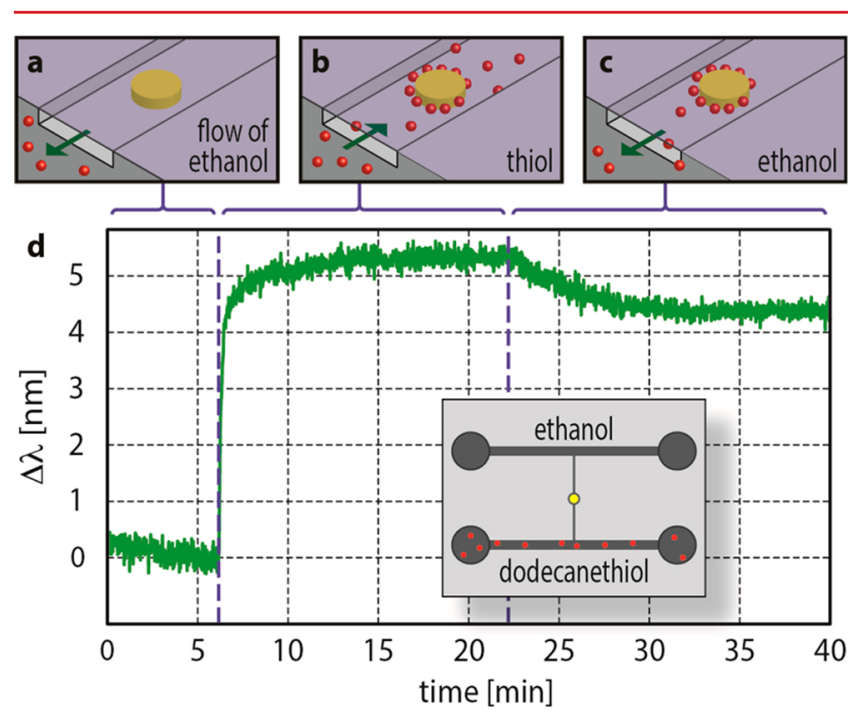

Figure 5. Dodecanethiol adsorption to a single Au nanoantenna in a single nanochannel. $(a-c)$ Schematic depictions of the different phases of the experiment, which was comprised of initial flushing of the nanochannel with ethanol (a), followed by flushing with dodecanethiol solution (b) and rinsing in pure ethanol (c). The arrows indicate the direction of the applied flows from the two connected microchannels acting as reservoirs for pure ethanol and dodecanethiol solution, respectively. (d) The time-dependent LSPR shift, $\Delta \lambda$ of an individual gold nanosensor in a nanochannel initially filled with ethanol upon exposure to $(50 \mathrm{mM})$ ethanol-based dodecanethiol solution, followed by a rinsing step in pure ethanol. The observed kinetics and the magnitude of the final $\Delta \lambda$-value compare well with reported LSPR shifts caused by thiol molecules binding to gold or silver nanoantennas of similar dimensions, and the finite $\Delta \lambda$-value upon rinsing shows the irreversible character of the binding process, as expected for a thiol.

traditional peak-shift readout scheme to facilitate direct comparison with existing literature. Figure $5 \mathrm{~d}$ shows the timedependent LSPR shift, $\Delta \lambda$, of an individual gold nanosensor in a nanochannel initially filled with ethanol. The nanochannel is then flushed with an ethanol-based $50 \mathrm{mM}$ dodecanethiol solution, and subsequently rinsed with ethanol. The observed kinetics, revealed by plotting the $\Delta \lambda$ signal as a function of time, are similar to reported binding kinetics of alkanethiols to open gold surfaces at high concentrations, ${ }^{45}$ and the magnitude of the final $\Delta \lambda$-value $(5.3 \mathrm{~nm})$ compares well with reported LSPR shifts caused by thiol molecules binding to gold or silver nanoantennas of similar dimensions. ${ }^{46,47}$ Our $\Delta \lambda$-value is slightly smaller because it is measured in solvent, which yields a smaller refractive index contrast compared to nitrogen gas usually used in the literature. Moreover, the finite $\Delta \lambda$-value upon rinsing shows the irreversible character of the binding process, as expected for a thiol.

In summary, we have implemented on-chip referenced single particle nanoplasmonic sensing in individual nanochannels by employing an advanced nanofabrication strategy that allows us to build nanofluidic devices with integrated plasmonic gold nanoantenna sensors with very high precision. We highlight that in such an arrangement it becomes possible to probe the entire volume of fluid flowing by the antenna, that is, any fluid and/or molecule flowing through the nanochannel must pass within the plasmonic field decay length, provided the gap between nanoantenna and channel wall is less than $\sim 30-50$ nm. ${ }^{33}$ The nanoantenna sensors can be individually addressed and read-off using both traditional polychromatic illumination and "peak shift" readout, as well as monochromatic laser illumination and single-wavelength scattering intensity readout. Moreover, on-chip referencing using a tailored "dead-end" nanochannel, which maintains constant conditions and prevents the reference nanoantenna from getting exposed to analyte during an experiment, makes it possible to eliminate unwanted signal drifts and artifacts caused by light source intensity fluctuations, and to obtain exceptional long-term stability of the single nanoantenna readout. The nanosensors inside the nanochannels generally obey the well-established size-dependent scaling of their bulk refractive index sensitivities as well as sensing FoM. However, as an additional important factor to consider, we find from our experiments, as well as from corresponding FDTD simulations, that the nanochannel width becomes important for the bulk sensing performance in the regime where the channel walls occupy a significant fraction of the sensing volume and thus reduce the bulk refractive index sensitivity. However, we also note that this effect most likely is negligible when molecular binding is to be detected.

We also demonstrated the attainable single nanoparticle sensing function inside nanofluidic structures on two specific examples: (i) in situ measurements of local buffer concentrations inside a nanofluidic channel at different applied conditions; (ii) real time binding kinetics of alkanetiol molecules to a single plasmonic nanonatenna sensor in a single nanochannel. The first example is important in light of that nanofluidic devices traditionally are used for studying individual biomolecules like DNA under different confinement conditions to correlate their properties with their local chemical and physical environment. However, to verify and locally assess the applied conditions (e.g., temperature, concentration of reagents, or ionic strength) inside the nanofluidic system in operando is to date one of the unsolved challenges in the field. Therefore, the presented solution may provide critical new insights in studies of individual biomolecules under extreme confinement. The second example provides a blueprint for how a nanofluidic system can be efficiently used to transport and direct analyte molecules toward a single plasmonic nanoantenna sensor, where they are detected upon binding. This opens up exciting new opportunities because by design essentially all analyte solution is forced to pass in extreme vicinity ${ }^{12}$ of the plasmonic nanoantenna sensor inside a single nanochannel.

In a long-term perspective, we envision our concept to provide a powerful solution for overcoming diffusion limitations ${ }^{32}$ in mass transport to and from individual plasmonic nanoparticles. This offers unique opportunities for label-free single molecule detection at low analyte concentrations. Specifically, we envision on-chip fluidic systems that are comprised of individually addressed nanofluidic structures, each decorated with a tailored single plasmonic antenna sensor, which spectroscopically are read off simultaneously. The individual plasmonic sensor nanoparticles, due to their material contrast with respect to the channel walls, can thus be individually functionalized with analyte-specific linkers through their respective nanofluidic system. Such a platform thus uniquely allows implementation of different functionalization tailored for different analytes on different nanoantenna sensors, and thus multiplexing at the single nanoparticle level. Moreover, our platform may serve as experimental tool for fundamental studies of fluids in extreme confinement, as well as 
for integration in lab-on-a-chip-type devices with volumes smaller than a single cell, ${ }^{12}$ functionalized with plasmonic sensing readout and/or signal enhancement functions such as enhanced fluorescence ${ }^{48}$ or surface-enhanced Raman scattering. $^{49}$

Methods. Nanofabrication. The fabrication process is summarized in Figure S3 in the SI and is described in full detail in the corresponding SI section. In short, it includes the following key steps: (a) definition and etching of nanochannels with electron beam lithography (EBL) and reactive-ion etching (RIE), respectively, into the surface of a thermally oxidized silicon wafer; (b) etching of microchannels connecting to the nanochannels with optical lithography (OL) and RIE; (c) etching of inlet holes with OL and deep reactive-ion etching (DRIE); (d) definition of plasmonic nanoantenna sensors with EBL and growth of the antennas via physical vapor deposition (PVD) through the EBL-mask, followed by lift-off; (e) selection of well-aligned sensors, fusion bonding of the nanostructured Si-wafer to a glass cover wafer; and dicing into fluidic chips. All fluid structures were created with reactiveion etching using fluorine-based chemistry. The lids (Pyrex, 175 $\mu \mathrm{m}$ thickness) were fusion-bonded to the substrates (silicon with $120 \mathrm{~nm}$ thermal oxide) for $5 \mathrm{~h}$ at $550{ }^{\circ} \mathrm{C}$.

Plasmonic Sensing Experiments. Single particle dark-field scattering measurements were carried out on a Nikon Eclipse LV100 upright optical microscope, using a $50 \mathrm{~W}$ halogen lamp (Nikon LV-HL50W LL) or a $20 \mathrm{~mW}$ HeNe laser (Thorlabs HNL210L) as illumination source. Rayleigh scattered light from the nanoantenna sensors was collected with a 50X objective (Nikon $50 \mathrm{BD}$ ) and directed to the entrance slit of a spectrometer (Andor Shamrock SR303i) via one broadband dielectric mirror and two visible achromats. The collected light was dispersed with a grating (150 lines $/ \mathrm{mm}$, blaze wavelength $800 \mathrm{~nm}$ ) onto the sensor of a thermoelectrically cooled CCD camera (Andor Newton 920). Note that the grating acts as a mirror when using monochromatic illumination. For experiments using white light and monochromatic illumination, the CCD camera was operated in full vertical binning mode and imaging mode, respectively.

Data Analysis. To account for both light scattering from the walls of nanochannels and spectral inhomogeneity of the light source, scattering spectra $I$ of the confined gold particles were determined according to $I=\left[I_{\mathrm{P}}-I_{\mathrm{B}}\right] / I_{\mathrm{L}}$, where $I_{\mathrm{P}}$ is the spectrum measured at the location of a particle, and $I_{\mathrm{B}}$ is an appropriate background spectrum. The spectrum of the illuminating lamp, $I_{\mathrm{L}}$, was recorded using the diffuse white certified reflectance standard Labsphere SRS-99-020. Background spectra $I_{\mathrm{B}}$ were taken of reference channels without plasmonic particles, obtained at the same distance from the microchannels as the studied particle. Values of LSPRs were determined by fitting second order polynomials $y(\lambda)=c_{1}+c_{2} \lambda$ $+c_{3} \lambda^{2}$ to the spectra $I(\lambda)$ of the confined gold particles, where $\lambda$ is the wavelength and $c_{1}$ through $c_{3}$ are fitting parameters.

FDTD Simulations. Simulations using the finite-difference time-domain (FDTD) method, carried out with the commercial software FDTD Solutions (Lumerical), were used to evaluate the electrodynamics of the experimental system. A gold sensor was simulated as a disk with dimensions tuned to match the resonance wavelength of the experiments $(d=80$ $\mathrm{nm}, h=30 \mathrm{~nm})$. The disk was placed inside a rectangular channel with a height of $30 \mathrm{~nm}$ and varying width, the material inside the channel was set as either water (refractive index of 1.333) or ethylene glycol (refractive index of 1.43) and the surrounding material was $\mathrm{SiO}_{2}$ (refractive index of 1.46) placed on top of $\mathrm{Si}$ (semi-infinite) with a channel-to-silicon distance of $100 \mathrm{~nm}$. $\mathrm{Au}$ and $\mathrm{Si}$ dielectric functions were taken from Johnson and Christy ${ }^{50}$ and Palik ${ }^{51}$ respectively. To correctly resolve the field close to the gold disk a mesh overlay with a step size of $0.5 \mathrm{~nm}$ was used around the disk. Light was introduced as a linearly polarized plane wave via a total-field/ scattered-field source and the scattering spectra were collected in the backward direction with respect to the incident field by integrating the Poynting vector of the scattered-field. A "dark" spectrum was simulated for each channel size by removing the Au disk, however, subtracting the "dark" from the "bright" spectra had only a negligible effect on the peak tracking because the bright spectra were about 1 order of magnitude larger compared to the dark. The field-enhancement plots were calculated as the enhanced field intensity divided by the incident field intensity.

\section{ASSOCIATED CONTENT}

\section{Supporting Information}

The Supporting Information is available free of charge on the ACS Publications website at DOI: 10.1021/acs.nanolett.6b04124.

Details on cross-sectional SEM imaging, device nanofabrication, raw data for bulk refractive index sensitivity, FoM analysis and FDTD simulations. (PDF)

\section{AUTHOR INFORMATION}

\section{Corresponding Authors}

*E-mail: fredrik.westerlund@chalmers.se.

*E-mail: clangham@chalmers.se.

ORCID

Tomasz J. Antosiewicz: 0000-0003-2535-4174

Christoph Langhammer: 0000-0003-2180-1379

\section{Author Contributions}

All authors have given approval to the final version of the manuscript.

\section{Notes}

The authors declare no competing financial interest.

\section{ACKNOWLEDGMENTS}

We acknowledge funding from the ERC Starting Grant SINCAT (C.L., D.A., J.F.), the Knut and Alice Wallenberg Foundation Grant 2015.0057 (C.L., J.F., F.W.), the Olle Engkvist Byggmästare Foundation, the Chalmers Area of Advance in Nanoscience and Nanotechnology (C.L., F.W.) and the Swedish Research Council Grant 2014-4956 (C.L.) and 2011-4324 (F.W.). We also thank the Knut and Alice Wallenberg Foundation for its support of the infrastructure in the MC2 nanofabrication laboratory at Chalmers, and the Swedish Research Council for its support of the $\mu$-fab cleanroom infrastructure in Sweden.

\section{REFERENCES}

(1) Bocquet, L.; Charlaix, E. Chem. Soc. Rev. 2010, 39, 1073-1095.

(2) Stein, D.; Kruithof, M.; Dekker, C. Phys. Rev. Lett. 2004, 93, 035901.

(3) Tsukahara, T.; Mawatari, K.; Hibara, A.; Kitamori, T. Anal. Bioanal. Chem. 2008, 391, 2745-2752.

(4) Zhang, J.; Todd, B. D.; Travis, K. P. J. Chem. Phys. 2004, 121, $10778-10786$.

(5) Israelachvili, J. N.; Pashley, R. M. Nature 1983, 306, 249-250. 
(6) Pu, Q.; Yun, J.; Temkin, H.; Liu, S. Nano Lett. 2004, 4, 10991103

(7) Persson, F.; Tegenfeldt, J. O. Chem. Soc. Rev. 2010, 39, 985-999.

(8) Arayanarakool, R.; Shui, L.; Kengen, S. W. M.; van den Berg, A.; Eijkel, J. C. T. Lab Chip 2013, 13, 1955-1962.

(9) Prakash, S.; Piruska, A.; Gatimu, E. N.; Bohn, P. W.; Sweedler, J. V.; Shannon, M. A. IEEE Sens. J. 2008, 8, 441-450.

(10) Wang, Y. M.; Tegenfeldt, J. O.; Reisner, W.; Riehn, R.; Guan, X.J.; Guo, L.; Golding, I.; Cox, E. C.; Sturm, J.; Austin, R. H. Proc. Natl. Acad. Sci. U. S. A. 2005, 102, 9796-9801.

(11) Wang, Y.-C.; Stevens, A. L.; Han, J. Anal. Chem. 2005, 77, 4293-4299.

(12) Shirai, K.; Mawatari, K.; Kitamori, T. Small 2014, 10, 15141522.

(13) Novotny, L.; Van Hulst, N. Nat. Photonics 2011, 5, 83-90.

(14) Anker, J. N.; Hall, W. P.; Lyandres, O.; Shah, N. C.; Zhao, J.; Van Duyne, R. P. Nat. Mater. 2008, 7, 442-453.

(15) Mayer, K. M.; Hafner, J. H. Chem. Rev. 2011, 111, 3828-3857.

(16) Tittl, A.; Giessen, H.; Liu, N. Nanophotonics 2014, 3, 157-180.

(17) Larsson, E. M.; Syrenova, S.; Langhammer, C. Nanophotonics 2012, 1, 249-266.

(18) Ringe, E.; Sharma, B.; Henry, A.-I.; Marks, L. D.; Van Duyne, R. P. Phys. Chem. Chem. Phys. 2013, 15, 4110-4129.

(19) Wang, W.; Tao, N. Anal. Chem. 2014, 86, 2-14.

(20) Zijlstra, P.; Orrit, M. Rep. Prog. Phys. 2011, 74, 106401.

(21) Guo, L.; Jackman, J. A.; Yang, H.-H.; Chen, P.; Cho, N.-J.; Kim, D.-H. Nano Today 2015, 10, 213-239.

(22) Sriram, M.; Zong, K.; Vivekchand, S.; Gooding, J. Sensors 2015, $15,25774$.

(23) Sannomiya, T.; Vörös, J. Trends Biotechnol. 2011, 29, 343-351.

(24) Holzmeister, P.; Acuna, G. P.; Grohmann, D.; Tinnefeld, P. Chem. Soc. Rev. 2014, 43, 1014-1028.

(25) Zijlstra, P.; Paulo, P. M. R.; Orrit, M. Nat. Nanotechnol. 2012, 7, $379-382$.

(26) Ament, I.; Prasad, J.; Henkel, A.; Schmachtel, S.; Sönnichsen, C. Nano Lett. 2012, 12 (2), 1092-1095.

(27) Beuwer, M. A.; Prins, M. W. J.; Zijlstra, P. Nano Lett. 2015, 15 (5), 3507-3511.

(28) Schuller, J. A.; Barnard, E. S.; Cai, W.; Jun, J. C.; White, J. S.; Brongersma, M. L. Nat. Mater. 2010, 9, 193-204.

(29) Xu, H.; Aizpurua, J.; Käll, M.; Apell, P. Phys. Rev. E: Stat. Phys., Plasmas, Fluids, Relat. Interdiscip. Top. 2000, 62, 4318-4324.

(30) Sheehan, P. E.; Whitman, L. J. Nano Lett. 2005, 5, 803-807.

(31) Nair, P. R.; Alam, M. A. Appl. Phys. Lett. 2006, 88, 233120.

(32) De Angelis, F.; Gentile, F.; Mecarini, F.; Das, G.; Moretti, M.; Candeloro, P.; Coluccio, M. L.; Cojoc, G.; Accardo, A.; Liberale, C.; et al. Nat. Photonics 2011, 5, 682-687.

(33) Dmitriev, A.; Hägglund, C.; Chen, S.; Fredriksson, H.; Pakizeh, T.; Käll, M.; Sutherland, D. S. Nano Lett. 2008, 8, 3893-3898.

(34) Hanarp, P.; Kall, M.; Sutherland, D. S. J. Phys. Chem. B 2003, 107, 5768-5772.

(35) Larsson, E. M.; Alegret, J.; Kall, M.; Sutherland, D. S. Nano Lett. 2007, 7, 1256-1263.

(36) Martinsson, E.; Shahjamali, M. M.; Large, N.; Zaraee, N.; Zhou, Y.; Schatz, G. C.; Mirkin, C. A.; Aili, D. Small 2016, 12, 330-342.

(37) Offermans, P.; Schaafsma, M. C.; Rodriguez, S. R. K.; Zhang, Y.; Crego-Calama, M.; Brongersma, S. H.; Rivas, J. G. ACS Nano 2011, 5, 5151-5157.

(38) Zoric, I.; Zäch, M.; Kasemo, B.; Langhammer, C. ACS Nano 2011, 5, 2535-2546.

(39) Wadell, C.; Nugroho, F. A. A.; Lidström, E.; Iandolo, B.; Wagner, J. B.; Langhammer, C. Nano Lett. 2015, 15, 3563-3570.

(40) Reisner, W.; Beech, J. P.; Larsen, N. B.; Flyvbjerg, H.; Kristensen, A.; Tegenfeldt, J. O. Phys. Rev. Lett. 2007, 99, 058302.

(41) Zhang, C.; Shao, P. G.; van Kan, J. A.; van der Maarel, J. R. C. Proc. Natl. Acad. Sci. U. S. A. 2009, 106, 16651-16656.

(42) Zhang, C.; Jiang, K.; Liu, F.; Doyle, P. S.; van Kan, J. A.; van der Maarel, J. R. Lab Chip 2013, 13, 2821-2826.

(43) Peacock, A. C.; Dingman, C. W. Biochemistry 1968, 7, 668-674.
(44) Syrenova, S.; Wadell, C.; Nugroho, F. A. A.; Gschneidtner, T. A.; Diaz Fernandez, Y. A.; Nalin, G.; Świtlik, D.; Westerlund, F.; Antosiewicz, T. J.; Zhdanov, V. P.; et al. Nat. Mater. 2015, 14, 12361244.

(45) Bain, C. D.; Barry Troughton, E.; Tao, Y. T.; Evall, J.; Whitesides, G. M.; Nuzzo, R. G. J. Am. Chem. Soc. 1989, 111, 321335.

(46) Rindzevicius, T.; Alaverdyan, Y.; Dahlin, A.; Höök, F.; Sutherland, D. S.; Käll, M. Nano Lett. 2005, 5, 2335-2339.

(47) Sherry, L. J.; Chang, S.-H.; Schatz, G. C.; Van Duyne, R. P.; Wiley, B. J.; Xia, Y. Nano Lett. 2005, 5, 2034-2038.

(48) Yuan, H.; Khatua, S.; Zijlstra, P.; Yorulmaz, M.; Orrit, M. Angew. Chem., Int. Ed. 2013, 52, 1217-1221.

(49) Qian, X. M.; Nie, S. M. Chem. Soc. Rev. 2008, 37, 912-920.

(50) Johnson, P. B.; Christy, R. W. Phys. Rev. B 1972, 6, 4370-4379.

(51) Palik, E. D. Handbook of Optical Constants of Solids; Academic Press: New York, 1997. 